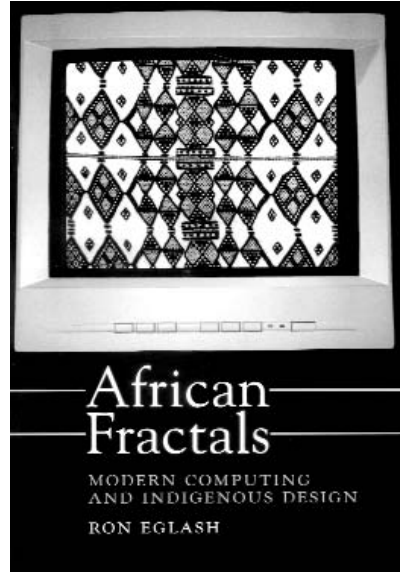

Ron Eglash

African Fractals: Modern Computing and Indigenous Design

New Brunswick NJ: Rutgers University Press, 1999

Reviewed by Abdul Karim Bangura

Every once in a while, a book is published that significantly advances scholarship. Thus, I am not going to be apologetic for beginning this review by stating boldly that Ron Eglash's African Fractals is bound to be regarded as one of the greatest books on African studies produced in this century. This brilliant, scientific work contributes significantly towards shattering long-held myths and misconceptions about Africans (both on the Continent and in the Diaspora), the most pervasive and pernicious of which is the notion of Africans as inactive agents in history a people devoid of writing systems, technological background and culture.

The book is divided into three parts with 14 chapters. The first part introduces fractal geometry for people without any mathematics background, fractals in African settlement architecture, fractals in cross-cultural comparison, and intention and invention in design. The second part discusses geometric algorithms, scaling, numeric systems, recursion, infinity, and complexity. The third part focuses on theoretical frameworks in cultural studies of knowledge, the politics of African fractals, fractals in European history of mathematics, and futures for African fractals.

What Eglash teaches in the 14 chapters is that elaborate cornrow braids on an African woman's head, for example, can be viewed as more than an affinity with culture or a fashion statement. The intricate patterns are also useful for learning about African fractals—geometric patterns that are repeated on smaller and smaller scales to produce intricate designs that are beyond the scope of classical or Euclidean geometry. Fractal geometry has emerged as one of the most exciting frontiers in the fusion between mathematics and information technology. Fractals can be observed in many of the swirling patterns produced by computer graphics, and they have become a vital tool for modeling in the natural sciences. While fractal geometry can allow one to get into the far reaches of high tech science, its patterns are surprisingly common in traditional African designs. Also, some of the basic concepts in fractal geometry are fundamental to African knowledge systems: quantitative techniques, symbolic systems, engineering, architecture, games, traditional hairstyling, textiles, sculpture, painting, carving, 
metalwork, and religion. As Eglash explains, although most people learn Euclidean geometry in school, few study fractal geometry, which plays a significant role in the computer modeling process in the hard sciences. Meanwhile, according to Eglash, fractal geometry has long been a theme in Africa, with a wide variety of local cultural associations, including kinship, labor practices, politics, and religion.

Eglash's research began in the 1980s while investigating settlement architecture in Central and West Africa. Aerial photographs of various settlement compounds revealed that many were composed of circular structures enclosed in other circles, or rectangles within rectangles, and that the compounds were likely to have street patterns in which broad avenues branched into very small footpaths. As Eglash notes, at first he thought it was just from unconscious social dynamics. But during his fieldwork, he found that fractal designs also appear in a wide variety of intentional designs - carving, hairstyling, metalwork, painting, textiles - and the recursive process of fractal algorithms are even employed in African quantitative systems. Eglash adds that in the design rationales and cultural semantics of many African geometric figures, as well as in indigenous quantitative systems (additive progression, doubling sequences, binary recursion) and symbolic systems (iconic symbols for feedback loops, equiangular spirals, infinity), there are abstract ideas and formal structures that closely parallel some of the fundamental aspects of fractal geometry. These results, Eglash concludes, are congruent with recent developments in complex systems theory, which suggest that premodern, non-state societies were neither utterly anarchic, nor frozen in static order, but rather utilized an adaptive flexibility that capitalized on the nonlinear aspects of ecological dynamics.

While in Africa, Eglash encountered some of the most complex fractal systems that exist in religious activities, such as the sequence of symbols used in sand divination, a method of fortune telling found in Senegal. Some of his other findings include the use of sophisticated mathematical ideas in everyday objects. In the arid region of the Sahel, for example, artisans produce windscreens by utilizing a scaling design that gives them the maximum effect -keeping out the wind-driven dust - for the minimum amount of effort and material.

When Eglash returned from Africa, one of his colleagues advised him to focus on scaling patterns in African hairstyles. An enthusiastic group of students at Evergreen State University volunteered their programming skills to help create a multimedia lesson on African fractals. The Hairstyle Storyboard Website (http://www.rpi.edu/ - eglash/eglash.dir/afmulti.htm) that has been developed utilizes a style referred to as "the braids of threads", from Yaoundé, Cameroon, to explicate African branching fractals. The "fractal hairstyle" module guides users, step by step, through the creation of a three-dimensional fractal, beginning with the initial design and then mathematically determining the ratio of each iteration. The major goal is to eventually combine the images, software and video on African fractals.

Given all this, at least two critical questions can be raised: (1) Since some scholars have found that all cities (historic, primitive and modern) are fractal precisely because they are complex natural systems, and other scholars have discovered that fractal tiling patterns exist on some of the oldest European tiled floors and in ancient Chinese art, what then does this say for the validity of Eglash's arguments concerning African fractals? (2) At what number of scales does self-similarity occur in African fractals and what method does Eglash employ to determine self-similarity? Eglash deals with these questions in several ways. 
First, Eglash demonstrates that traditional African settlements typically show repetition of similar patterns at ever-diminishing scales: circles of circles of circular dwellings, rectangular walls enclosing ever-smaller rectangles, and streets in which broad avenues branch down to tiny footpaths with striking geometric repetition. He easily identifies the fractal structure when he compares aerial views of African villages and cities with corresponding fractal graphics simulations. To estimate the fractal dimension of a spatial pattern, Eglash uses several different approaches. In the case of Mokoulek, for instance, which is a black-and-white architectural diagram, a twodimensional version of the ruler size versus length plots is employed. However, for the aerial photo of Labbazanga, an image in shades of gray, a Fourier transform is used. Nonetheless, according to Eglash, we cannot just assume that African fractals show an understanding of fractal geometry, nor can we dismiss that possibility. Thus, he insists that we listen to what the designers and users of these structures have to say about it. This is because what may appear to be an unconscious or accidental pattern might actually have an intentional mathematical component.

Second, as Eglash examines African designs and knowledge systems, five essential components (recursion, scaling, self-similarity, infinity, and fractional dimension) keep him on track of what does or does not match fractal geometry. Since scaling and self-similarity are descriptive characteristics, his first step is to look for the properties in African designs. Once he establishes that theme, he then asks whether or not these concepts have been intentionally applied, and starts to look for the other three essential components. He finds the clearest illustrations of indigenous self-similar designs in African architecture.

The examples of scaling designs Eglash provides vary greatly in purpose, pattern, and method. As he explains, while it is not difficult to invent explanations based on unconscious social forces - for example, the flexibility in conforming designs to material surfaces as expressions of social flexibility - he does not believe that any such explanation can account for its diversity. He finds that from optimization engineering, to modeling organic life, to mapping between different spatial structures, African artisans have developed a wide range of tools, techniques, and design practices based on the conscious application of scaling geometry. Thus, for example, instead of using the Koch curve to generate the branching fractals used to model the lungs and acacia tree, Eglash uses passive lines that are just carried through the iterations without change, in addition to active lines that create a growing tip by the usual recursive replacement.

In essence, any serious student of African studies, anthropology, computer science, engineering, or mathematics should run to a bookstore and get a copy of Eglash's unique masterpiece. It is worth every penny. As a matter of fact, the book has motivated me to push on with my own work entitled Chaos Theory and African Fractals. I am quite sure that some readers will be stimulated in similar ways.

First published in the NNJ online October 2000

\section{The Reviewer}

Abdul Karim Bangura is currently Director of The African Institution in Washington, DC; Project Co-director of The International Communication and Negotiation Simulations Project at the University of Maryland at College Park; Researcher-In-Residence at the Center of Global Peace and Professorial Lecturer in International 
Peace and Conflict Resolution in the School of International Service at American University in Washington, DC; and Professorial Lecturer in Political Science at Howard University in Washington, DC. Bangura holds three Ph.Ds to date-in Political Science from Howard University, in Policy Sciences (specializing in Development Economics) from the University of Maryland Baltimore, and in Linguistics from Georgetown University - and is completing a fourth Ph.D. in Computer Science at Columbus University. He is the author of 19 books and more than 100 articles in refereed journals and other sources. His recent publications include Chaos Theory and African Fractals (in preparation) and Historical Political Economy of Washington, DC (2000). He is the Editor-In-Chief of two refereed journals: Journal of Research Methodology and African Studies and African Journal of Languages and Linguistics. He is a member of and holds offices in many professional associations. He also is fluent in about a dozen African and six European languages. 